\title{
Canonical Least-Squares Monte Carlo Valuation of American Options: Convergence and Empirical Pricing Analysis
}

\author{
Xisheng Y $\mathbf{u}^{1}$ and Qiang Liư ${ }^{2}$ \\ ${ }^{1}$ School of Economic Mathematics, Southwestern University of Finance and Economics, 555 Liutai Boulevard, \\ Wenjiang, Chengdu 611130, China \\ ${ }^{2}$ School of Finance, Southwestern University of Finance and Economics, Chengdu 610074, China
}

Correspondence should be addressed to Xisheng Yu; yuxisheng@swufe.edu.cn

Received 5 January 2014; Revised 7 May 2014; Accepted 11 May 2014; Published 3 June 2014

Academic Editor: Wei-guo Zhang

Copyright (C) 2014 X. Yu and Q. Liu. This is an open access article distributed under the Creative Commons Attribution License, which permits unrestricted use, distribution, and reproduction in any medium, provided the original work is properly cited.

\begin{abstract}
The paper by Liu (2010) introduces a method termed the canonical least-squares Monte Carlo (CLM) which combines a martingaleconstrained entropy model and a least-squares Monte Carlo algorithm to price American options. In this paper, we first provide the convergence results of CLM and numerically examine the convergence properties. Then, the comparative analysis is empirically conducted using a large sample of the S\&P 100 Index (OEX) puts and IBM puts. The results on the convergence show that choosing the shifted Legendre polynomials with four regressors is more appropriate considering the pricing accuracy and the computational cost. With this choice, CLM method is empirically demonstrated to be superior to the benchmark methods of binominal tree and finite difference with historical volatilities.
\end{abstract}

\section{Introduction}

Stutzer [1] has initially proposed the canonical valuation method for valuing European options under an entropy framework and due to its effective performance in pricing European options, the canonical valuation was extended to value American options by employing the least-squares Monte Carlo algorithm (LSM, see [2-5]). However, neither of them is analyzed on the convergence and no empirical investigation is done on the former approach termed canonical least-squares Monte Carlo (CLM) method. This paper sets out to analyze the convergence properties for the CLM method and to document the empirical performance of the CLM method.

As is known, the crucial part of the LSM approach is the approximation of a set of conditional expectation functions and exactly due to this, it is necessary to explore the convergence for the cross-sectional regressions and for the estimated price. Clément et al. [6] prove that, for any fixed number of basis functions, the conditional expectation functions can be reached as the number of simulated paths tends to infinity and then converges to the value function of American option as the number of regressors tends to infinity. A further study in Stentoft [7] provides the rate of convergence of the conditional expectation approximation to the true expectation function as well as that of the American price estimate to the true price.

For the newly proposed CLM method, having generated the canonical risk-neutral paths of the underlying price, it uses the LSM algorithm to determine the optimal stopping rule along each of the generated paths so as to ultimately produce the price of the American option. Therefore, the convergence of CLM method is more like that of LSM method. And for the purpose of examining the convergence, in this paper we conduct a numerical analysis by varying the number of the paths and the number of regressors as well as the type of regressors, each of which could influence the performance of the method and hence affect the results obtained.

It needs to be noted that, to obtain the rate of convergence, this paper slightly modifies the CLM in the following way: when approximating the conditional expectation function, a new set of independently simulated paths is used for the 
regressions at each time step, rather than using the same set of paths at all the time points. See Section 3.1 for details.

Another purpose of this paper is to test the pricing performance of the CLM method. Following a general tradition (as the typical representatives of index option and equity option, S\&P 100 Index and IBM options are widely used in many empirical studies when one new method is proposed. For example, Harvey and Whaley $[8,9]$ are the first to treat the S\&P 100 Index options as American-style options in their empirical studies. The effect of stochastic dividends and volatility on the price and exercise boundary of the American-style S\&P 100 Index calls was studied by Broadie et al. [10], who also investigated empirically the Americanstyle S\&P 100 Index options [11]. The S\&P 100 Index options were also used in those studies [12-16]. For equity options, American put options on four common stocks, including IBM, were studied using the neural network approach by Kelly and Shorish [17]. Stentoft [18] used IBM puts among others to test his option pricing method based on GARCH models and the normal inverse Gaussian distribution), we choose the American-style S\&P 100 Index (OEX) (OEX is the ticker symbol for options on the S\&P 100 Index with American-style exercise and expiration on the third Friday. In addition, there are weekly OEX Options that expire within several weeks but not on the third Friday, and OEX LEAPS Options that last for two to three years and expire on the third Friday. Further, CBOE offers XEO, the European-style options on the S\&P 100 Index) puts and IBM puts for the empirical investigation. Unlike previous empirical work, the current study seems to be also notable in four aspects: (1) data of both IBM puts and OEX puts for a period which covers the peak period of the 2008 US financial crisis are collected; (2) all possible moneyness (here moneyness for puts is defined as the ratio of the strike price to the underlying asset's price) are considered; (3) dividend, an important factor for Americanstyle options $[8,10]$, is treated explicitly; (4) the risk-free interest rate utilized is interpolated linearly from the daily US Treasury yield curve. Meanwhile, CLM generates simulated paths for the underlying process from a canonical riskneutral distribution, while Alcock-Carmichael [4] simulates underlying paths directly from historical gross returns. As a consequence, CLM can work with a much smaller set of historical gross returns in obtaining the canonical distribution and in simulating paths. In addition, when deriving the distributions, we use the previous 130 or 260 daily gross returns, so are the paths simulated. The pricing results are collected into twelve categories of moneyness maturity and compared to those of the benchmark methods of binomial tree and finite difference by using three well-known error statistics. Overall, the CLM is found to be superior to the benchmarks.

The remainder of the paper is constructed as follows. Section 2 introduces the CLM valuation framework with detailed procedures. Section 3 discusses the convergence properties of CLM and numerically examines the pricing accuracy. Section 4 describes the methodology and the data of our empirical examination as well as the treatment of the important factors of dividend and interest rate. Also in this section the results of our empirical study are reported. Section 5 concludes.

\section{Canonical Least-Squares Monte Carlo Valuation of American Options}

2.1. Canonical Risk-Neutral Distribution. Initially the task of CLM valuation is to derive the "best" equivalent martingale measure $\pi^{*}$, for the daily gross return $R$ of the underlying asset, from a prior empirical probability distribution. Here we use the principle of maximum entropy. Entropy is an information theoretic concept that can be crudely interpreted as "explanatory information contained within a sample." When sample data, say a sample of historical gross returns, is transformed, much of the explanatory information contained in the sample is lost due to the transformation process. Within the context of entropy, the "best" measure transformation is that which loses the least entropy during transformation.

Assume a sample of $n$ observations of historical daily price ratios (or gross returns) of the underlying asset, $\left\{R_{k}=\right.$ $\left.S_{k-1} / S_{k}: k=1,2, \ldots, n\right\}$, where $S_{k}$ denotes that the $k$ th historical underlying price is given and the continuously compounded risk-free interest rate is $r$ (the risk-free interest rate varies from the trading date and the time to maturity. Its treatment is discussed in Section 4.2). Denote the riskneutral (martingale) probability of $R_{k}$ by $\pi_{k}^{*}$ that is to be determined. The entropy pricing framework is given as follows:

$$
\begin{aligned}
& \hat{\pi}^{*}= \underset{\pi_{k}^{*}>0, \sum_{k=1}^{n} \pi_{k}^{*}=1}{\arg } \min \sum_{k=1}^{n} \pi_{k}^{*} \log \left(\pi_{k}^{*}\right), \\
& \text { s.t. } \quad E_{\pi^{*}}(R)=\exp \left(\frac{r}{365}\right),
\end{aligned}
$$

where the constraint equation $E_{\pi^{*}}(R)=\exp (r / 365)$ is the martingale restriction and exactly the expectation under the martingale measure $\pi^{*}$ can be expressed as follows:

$$
E_{\pi^{*}}(R)=\sum_{k=1}^{n} \pi_{k}^{*} R_{k}
$$

This constrained minimization (1) can be solved using the Duality Theorem (see, e.g., Theorem 1 in [19, pages 264, 268-269]) by constructing the Lagrangian function. Consequently, the resultant risk-neutral martingale probability is

$$
\pi_{k}^{*}=\frac{\exp \left(\gamma^{*}\left(R_{k} / \exp (r / 365)\right)\right)}{\sum_{k=1}^{n} \exp \left(\gamma^{*}\left(R_{k} / \exp (r / 365)\right)\right)}, \quad k=1,2, \ldots, n,
$$

where $\gamma^{*}$ is the Lagrangian multiplier and can be obtained by solving the following unconstrained problem:

$$
\gamma^{*}=\underset{\gamma}{\arg \min } \sum_{k=1}^{n} \exp \left[\gamma\left(\frac{R_{k}}{\exp (r / 365)}-1\right)\right] .
$$

Thus $\pi_{k}^{*}$ in (3) provides a risk-neutral distribution for the daily underlying gross return and is termed here the canonical risk-neutral distribution from which independent random samples of gross returns can be drawn and hence the underlying price paths generated. It should be noted that although the canonical risk-neutral distribution we derived is for daily gross return $R_{k}$, it is exactly applicable to any interval of length of gross return if $1 / 365$ in (3) is replaced with the length of such interval. 
2.2. Risk-Neutral Paths of the Underlying Price. According to the risk-neutral distribution $\pi_{k}^{*}$ given in (3), the independent random sample of future gross returns can be drawn from the set of historical gross returns. To be specific, starting with the valuation date $t_{0}$, each historical return $R_{k}$ is associated with a risk-neutral probability $\pi_{k}^{*}$ and a sample of $n$ daily returns $\left\{\widetilde{R}_{1}, \widetilde{R}_{2}, \ldots, \widetilde{R}_{n}\right\}$ is randomly drawn from the set $\left\{R_{k}, k=1,2, \ldots, n\right\}$ according to $\pi_{k}^{*}$ employing the inverse transform method (Brandimarte [20, pages 230-232]). With the sampled returns and the initial price $S_{t_{0}}$ given, a riskneutral price path is then generated. We repeat this procedure $N$ times and obtain $N$ risk-neutral price paths as follows:

$$
\begin{aligned}
S_{t_{1}}^{(k)} & =S_{t_{0}} \widetilde{R}_{1}^{(k)}, \quad S_{t_{2}}^{(k)}=S_{t_{1}}^{(k)} \widetilde{R}_{2}^{(k)} \\
& =S_{t_{0}} \widetilde{R}_{1}^{(k)} \widetilde{R}_{2}^{(k)}, \ldots, S_{n}^{(k)}=S_{t_{0}} \prod_{i=1}^{n} \widetilde{R}_{i}^{(k)} \quad(k=1,2, \ldots, N),
\end{aligned}
$$

where $\widetilde{R}_{i}^{(k)}$ is the $i$ th random sample of the gross return along the $k$ th underlying path (note that, in this paper, such generated price paths are only used at one time step. To achieve the rate of convergence, the procedure needs to be independently repeated $K$ (the number of potential exercise points) times. The reason for this is that the same set of simulated paths used at each step could result in the dependence between the payoff paths. However, the paths should be asymptotically independent since the conditional expectation approximation converges to the true function. This issue is discussed in Section 3.1).

Now with those generated risk-neutral price paths, the LSM algorithm of Longstaff and Schwartz [2] is then applied to determine the optimal stopping strategy for each path and hence the American option can be valued by discounting each cash flow at the optimal exercise points back to time zero and taking the average over all the paths.

2.3. Optimal Stopping Strategy Based on Least-Squares Algorithm and the American Option Value. This subsection considers the American option valuation as an optimal stopping time problem (alternatively one can treat it as a problem of "value function" by defining the value of the option at potential exercise time $t_{k}, V\left(t_{k}\right)=\max \left(Z\left(t_{k}\right), E_{\pi^{*}}\left[V\left(t_{k+1}\right)\right.\right.$ | $\left.S\left(t_{k}\right)\right]$ ), and this can be solved by another algorithm not given here) so as to use the least-squares algorithm to determine the optimal stopping strategy. To fix notation, we let $t_{0}=0$, $t_{K}=T$, and $r$ denote the valuation date, maturity date, and the risk-free interest rate, respectively, and assume that an American option can only be exercised at a set of $K$ fixed time points $0=t_{0}<t_{1}<t_{2}<\cdots<t_{K}=T$. In addition, $\omega$ denotes a sample path in the Monte Carlo simulation (or specifically $\omega_{n}$ stands for the $n$th path, $\left.0 \leq n \leq N\right)$ and $S\left(\omega, t_{k}\right)$ is the underlying price at time $t_{k}$ along the path of $\omega$. We further denote by $Z\left(\omega, t_{k}\right)$ an adapted payoff process; for example, for the American put option with strike price $K$, $Z\left(\omega, t_{k}\right)=\max \left(K-S\left(\omega, t_{k}\right), 0\right)$. Finally we let $T\left(t_{k}\right)$ denote the set of all stopping times with values in $\left\{t_{k}, \ldots, t_{K}\right\}$ and define the function $C\left(\omega, \tilde{\tau}\left(t_{k}\right)\right)=e^{-r\left(\widetilde{\tau}\left(t_{k}\right)-t_{k}\right)} Z\left(\omega, \widetilde{\tau}\left(t_{k}\right)\right)$ as the cash flow of an option discounted back to $t_{k}$ conditional on the option not being exercised up to time and on following a stopping strategy, written as $\tilde{\tau}\left(t_{k}\right)$, from $t_{k}$ to $T$.

With these notations we can write the price of an American option as follows:

$$
V(0)=\max _{\tilde{\tau}(0) \in T(0)} E_{\pi^{*}}[C(\omega, \tilde{\tau}(0))],
$$

where the maximization is over stopping times $\tilde{\tau}(0) \in T(0)$ and the expectation is under the risk-neutral measure $\pi^{*}=$ $\left(\pi_{1}^{*}, \pi_{2}^{*}, \ldots, \pi_{n}^{*}\right)$ in $(3)$.

For the American option pricing problem (6), the optimal stopping times $\tau\left(t_{k}\right)$ can be generated iteratively according to the following algorithm:

$$
\begin{gathered}
\tau\left(t_{K}\right)=t_{K}=T, \\
\tau\left(t_{k}\right)=t_{k} 1_{\left\{Z\left(\omega, t_{k}\right) \geq E_{\pi^{*}}\left[C\left(\omega, \tau\left(t_{k+1}\right)\right) \mid S\left(\omega, t_{k}\right)\right]\right\}} \\
+\tau\left(t_{k+1}\right) 1_{\left\{Z\left(\omega, t_{k}\right)<E_{\pi^{*}}\left[C\left(\omega, \tau\left(t_{k+1}\right)\right) \mid S\left(\omega, t_{k}\right)\right]\right\}}, \\
k \leq K-1 .
\end{gathered}
$$

Obviously, if the conditional expectations given by $E_{\pi^{*}}\left[C\left(\omega, \tau\left(t_{k+1}\right)\right) \quad \mid \quad S\left(\omega, t_{k}\right)\right]$ are known, then the value of the option in (6) can be expressed in terms of the optimal stopping times in (7) as follows:

$$
V(0)=E_{\pi^{*}}[C(\omega, \tau(0))] .
$$

Although the conditional expectation function $E_{\pi^{*}}\left[C\left(\omega, \tau\left(t_{k+1}\right)\right) \mid S\left(\omega, t_{k}\right)\right]$ cannot be calculated, as those future cash flows are in general not known at time $t_{k}$, LSM bypasses this problem by working backwards and approximating this function, here we write it as $H\left(\omega, t_{k}\right) \equiv E_{\pi^{*}}\left[C\left(\omega, \tau\left(\omega, t_{k+1}\right)\right) \mid S\left(\omega, t_{k}\right)\right]$, by a linear sum based on $M$ terms

$$
H_{M}\left(\omega, t_{k}\right) \equiv \sum_{m=0}^{M-1} a_{m}\left(t_{k}\right) \phi_{m}\left(S\left(\omega, t_{k}\right)\right),
$$

where $\left\{\phi_{m}(\cdot)\right\}_{m=0}^{M-1}$ form a basis such as Monomials, Laguerre, and Shifted Legendre polynomials. However, the coefficients $\left\{a_{m}\left(t_{k}\right)\right\}_{m=0}^{M-1}$ at time $t_{k}$ are generally unknown and need to be estimated by a coefficient series, written as $\left\{\widetilde{a}_{m}^{N}\left(t_{k}\right)\right\}_{m=0}^{M-1}$, using the least-squares regression when implementing the procedure, where the coefficients $\left\{\widetilde{a}_{m}^{N}\left(t_{k}\right)\right\}_{m=0}^{M-1}$ are calculated as the solution to the following minimization problem:

$$
\begin{aligned}
\min _{\left\{\widetilde{a}_{m}^{N}\right\}_{m}} \sum_{n=1}^{N}\left[\sum_{m=0}^{M-1} \tilde{a}_{M}^{N}\left(t_{k}\right) \phi_{m}\left(S\left(\omega_{n}, t_{k}\right)\right)\right. \\
\left.\quad-e^{-r\left(t_{k+1}-t_{k}\right)} C\left(\omega_{n}, \widetilde{\tau}_{M}^{N}\left(t_{k+1}\right)\right)\right]^{2} .
\end{aligned}
$$

From this, an approximation $\widetilde{H}_{M}^{N}\left(\omega, t_{k}\right)$ to $H\left(\omega, t_{k}\right)$ can be constructed as follows:

$$
\widetilde{H}_{M}^{N}\left(\omega, t_{k}\right)=\sum_{m=0}^{M-1} \widetilde{a}_{m}^{N}\left(t_{k}\right) \phi_{m}\left(S\left(\omega, t_{k}\right)\right) .
$$


Then an approximation $\widetilde{\tau}_{M}^{N}\left(t_{k}\right)$ to the optimal stopping time algorithm $\tau\left(t_{k}\right)$ in (7) can be derived as follows:

$$
\begin{aligned}
& \widetilde{\tau}_{M}^{N}\left(t_{K}\right)=t_{K}=T, \\
& \widetilde{\tau}_{M}^{N}\left(t_{k}\right)=t_{k} 1_{\left\{Z\left(\omega, t_{k}\right) \geq \widetilde{H}_{M}^{N}\left(\omega, t_{k}\right)\right\}} \\
& \quad+\widetilde{\tau}_{M}^{N}\left(t_{k+1}\right) 1_{\left\{Z\left(\omega, t_{k}\right)<\widetilde{H}_{M}^{N}\left(\omega, t_{k}\right)\right\}}, \quad k \leq K-1,
\end{aligned}
$$

where $1_{\{\cdot\}}$ is the indicator function.

Using (10) and (11) yields the best linear approximation to $H_{M}\left(\omega, t_{k}\right)$ based on the $N$ simulated paths and then the option value in (8) can be estimated as follows:

$$
V_{M}^{N}(0)=\frac{1}{N} \sum_{n=1}^{N} C\left(\omega_{n}, \widetilde{\tau}_{M}^{N}(0)\right) .
$$

\section{Convergence of Canonical Least-Squares Monte Carlo}

As described in Section 2, having simulated the risk-neutral paths for the underlying, the CLM method employs the least-squares regression to determine the optimal stopping strategy. Therefore the convergence of CLM is dependent on the least-squares algorithm. This section presents the convergence results and conducts numerical simulations to examine the convergence properties.

3.1. Convergence Results. From (13), it seems reasonable that the estimated price converges to the true price of the American option if the approximation of the conditional expectation converges to the true expectation function. The following proposition verifies this with the proof of which can be found in [7].

Proposition 1. Assume that (i) the simulated underlying paths are independent and (ii) $\operatorname{Pr}\left(Z\left(\omega, t_{k}\right)=H\left(\omega, t_{k}\right)\right)=0,(0 \leq k \leq$ $K)$. Then $V_{M}^{N}(0) \rightarrow V(0)$ in probability, if $\widetilde{a}_{M}^{N}(0) \rightarrow a(0)$ as $N \rightarrow \infty$.

This proposition allows us to focus on the convergence of the conditional expectation approximations when examining the convergence of the algorithm. Below we provide a convergence theorem for the conditional expectation approximation in LSM algorithm.

Theorem 2. If $M=M(N)$ is increasing in $N$ such that $M \rightarrow$ $\infty$ and $M^{3} / N \rightarrow \infty$ as $N \rightarrow \infty$, then $\widetilde{H}_{M}^{N}\left(\omega, t_{k}\right)$ converges to $H\left(\omega, t_{k}\right)$ in probability for $k=1,2, \ldots, K$.

Proof. See Stentoft [7] for the proof and the necessary assumptions.

Together with Proposition 1, this theorem shows that the LSM price estimate converges to the true price under certain regularity assumptions when $M$ (as a function of $N$ ) tends to infinity but $M^{3} / N$ tends to zero as $N$ tends to infinity. It should be noted that Theorem 2 clearly shows that both the number of paths and the number of regressors should tend to infinity to achieve convergence of the American option price estimate from the LSM method. Furthermore, an essential requirement is that the speed with which the number of regressors increases is not so fast since $M^{3} / N \rightarrow 0$. In this paper we choose $M \propto N^{1 / 4}\left(M=0.4 N^{1 / 4}\right)$ when implementing CLM method in Section 4.

On the rate of convergence for such method based on least-squares regression, Stentoft [7] provides a result for convergence of the conditional expectation approximation in a two-period setting, stated as below.

Theorem 3. If $M=M(N)$ is increasing in $N$ such that $M \rightarrow \infty$ and $M^{3} / N \rightarrow \infty$ as $N \rightarrow \infty$, then the estimator $\widetilde{H}_{M}^{N}(\omega)$ is mean square convergent to $H\left(\omega, t_{k}\right)$ with $\int\left(H(\omega)-\widetilde{H}_{M}^{N}(\omega)\right)^{2} d F(s)=O_{p}\left(M / N+M^{-2 d}\right)$, where $F(s)$ denotes the cumulative distribution function of $s$ and $d$ is the number of continuous derivatives of the conditional expectation function that exits.

From this theorem, the optimal rate of convergence can be obtained as long as both $M / N$ and $M^{-2 d}$ have the same rate of convergence (i.e., $M$ is proportional to $N^{1 /(1+2 d)}$ ) for the LSM algorithm in the two-period setting. The optimal rate is then given by $N^{-2 d /(1+2 d)}$. In a multiperiod setting, however, the rate of convergence may be difficult to achieve due to the dependence between the payoff paths in the regressions. Thus to obtain such a rate, a new set of independently simulated paths is used for the regressions at each time step so as to allow us to use Theorem 3, but obviously this increases the computational cost.

3.2. Numerical Assessing. From (12) and the resulting option price formula in (13), it is obvious that the estimate depends on the number of regressors used in the cross-sectional regressions, $M$, as well as the number of paths used in the simulations, $N$. Furthermore, the convergence theorems above show that both the number of paths and the number of regressors should tend to infinity in order to estimate the conditional expectation arbitrarily well. In this section, prior to the empirical examination of CLM method, we conduct some numerical tests using a number of artificial put options under the Black-Scholes framework and report the results for the estimated conditional expectations and for the estimated option prices as the number of regressors, $M$, and the number of paths, $N$, increase.

3.2.1. Initial Setting. To provide a reference for the empirical study in Section 4, the numerical tests here use two sets of parameter values as given in Table 1, which match the corresponding values used in the empirical study when pricing IBM and OEX put options. To be specific, in the empirical section, when pricing an IBM put option traded on October 17, 2008, we use the parameter values with stock price being 88.91, strike price 90, time to maturity 182 days, historical volatility $27.78 \%$, and interest rate $1.27 \%$; when pricing an OEX put traded on January 16, 2009, the parameter values are stock index price of 400.96 , strike price 400, time to maturity 336 days, historical volatility $37.89 \%$, 
TABLE 1: Two sets of parameter values in the numerical tests.

\begin{tabular}{lccccc}
\hline & Initial price & Strike price & Maturity date & \multicolumn{2}{c}{ Interest rate } \\
& $S_{0}$ & $K$ & $T$ & $r$ & \multicolumn{2}{c}{ Volatility } \\
\hline First & 89 & 90 & 0.5 & $1.3 \%$ & $28 \%$ \\
Second & 401 & 400 & 1.0 & $0.4 \%$ & $38 \%$ \\
\hline
\end{tabular}

Note. This table gives two sets of parameter values for corresponding American put options. Both underlying assets are assumed to pay no dividends and the trading date $t_{0}$ is set to be time 0 so that the time to maturity is $T$.

TABLE 2: Polynomial families used in the numerical experiment.

\begin{tabular}{lcccc}
\hline & Constant term & First term & Second term & $n$th term \\
\hline Monomials & $M_{0}(x)=1$ & $M_{1}(x)=x$ & $M_{2}(x)=x^{2}$ & $M_{n}(x)=x^{n}$ \\
Weighted Laguerre & $W_{0}(x)=1$ & $W_{1}(x)=1-x$ & $W_{2}(x)=\frac{1}{2 !}\left(x^{2}-4 x+2\right)$ & $W_{n}(x)=\frac{e^{x}}{n !} \frac{d^{n}}{x^{n}}\left(x^{n} e^{-x}\right)$ \\
Shifted Legendre & $S_{0}(x)=1$ & $S_{1}(x)=2(2 x-1)$ & $S_{2}(x)=4\left(6 x^{2}-6 x+1\right)$ & $S_{n}(x)=\frac{(-1)^{x}}{2^{n} n !} \frac{d^{n}}{x^{n}}\left(\left[1-(2 x-1)^{2}\right]^{n}\right)$ \\
\hline
\end{tabular}

Note. This table gives three orthogonal polynomial families. The first three polynomials plus the constant term of each family are used in this numerical experiment.

and interest rate $0.41 \%$. This section is just to numerically examine the convergence; so for simplicity, we assume that the underlying asset pays no dividends and is traded at time 0 , and we conduct this simulation test under the Black-Scholes framework as did in $[2,3]$.

To simulate the risk-neutral paths of the underlying asset, we assume a geometric Brownian motion (GBM) for the underlying price process (the drift rate is set to be risk-free interest rate; hence the resultant underlying asset price is riskneutral). Consider

$$
d S(t)=r S(t)+\sigma S(t) d B(t)
$$

where $r$ is the risk-free interest rate, $\sigma$ the constant volatility, and $B(t)$ a standard Brownian motion. The well-known solution of the above GBM is

$$
S(t)=S_{0} \exp \left\{\frac{r-\sigma^{2}}{2} t+\sigma B(t)\right\},
$$

which can be discretized as a sequence of values at the potential exercise points $0=t_{0}<t_{1}<\cdots<t_{K}=T$ :

$$
S\left(t_{i}\right)=S_{i-1} \exp \left\{\frac{r-\sigma^{2}}{2}\left(t_{i}-t_{i-1}\right)+\sigma \sqrt{t_{i}-t_{i-1}} \varepsilon_{i}\right\},
$$

where $\varepsilon_{i}$ indicates the $i$ th random draw from the standard normal distribution.

Following formula (16), $N$ number of underlying price paths are generated at each time step for the regressions; thus totally $K \times N$ prices are simulated. With these risk-neutral paths, the optimal stopping strategy can then be determined so that the option value can be calculated as described in Section 2.3.

The convergence results in Section 3.1 guarantee the convergence of estimated price to the true price and show that the number of regressors used in the cross-sectional regressions, $M$, and the number of paths used in the simulations, $N$, have an influence on the convergence results. In addition, the choice of the type of regressors is also of importance. To investigate the convergence results, several numerical tests are conducted with different number of paths and regressors and different type of regressors. This investigation provides a reference for the empirical pricing using CLM. We here use the prices obtained from binomial tree method as the "true" prices of the American puts. In this binomial model, the up and down factors are calculated by $u=e^{\sigma \sqrt{\Delta t}}$ and $d=e^{-\sigma \sqrt{\Delta t}}$ where $\sigma$ represents the volatility given in Table 1 and $\Delta t$ is the length of time interval of one step. We use 8,000-period binomial tree and 4,000-period tree, respectively, for pricing the first put option and the second put.

The numerical tests vary the number of regressors, $M$, from 2 to 5 and the number of simulated (risk-neutral) paths, $N$, from 5,000 to 30,000 . Meanwhile we also use three types of polynomial families for the regressions in the numerical experiment: Monomials, weighted Laguerre polynomials, and Shifted Legendre polynomials which are listed in Table 2, where the elements of the Laguerre family have the property of being mutually orthogonal with respect to the weighting function $\omega(x)=e^{-x}$, and the Shifted Legendre polynomials have another advantage of no computationally intensive weights having to be calculated since the weighting function $\omega(x)=1$.

3.2.2. Result Analysis. The benchmark with which we compare, as mentioned earlier, is the binomial tree method, and the parameters of two American put options are given in Table 1. The results for American puts with various combinations of number of paths, number of regressors, and type of regressors are reported in Table 3.

From Table 3, several observations can be made. First of all, compared with the binomial tree method, the leastsquares algorithm CLM method used produces a negative pricing bias at most of all the combinations with only four exceptions. It is understandable for this finding, since the 


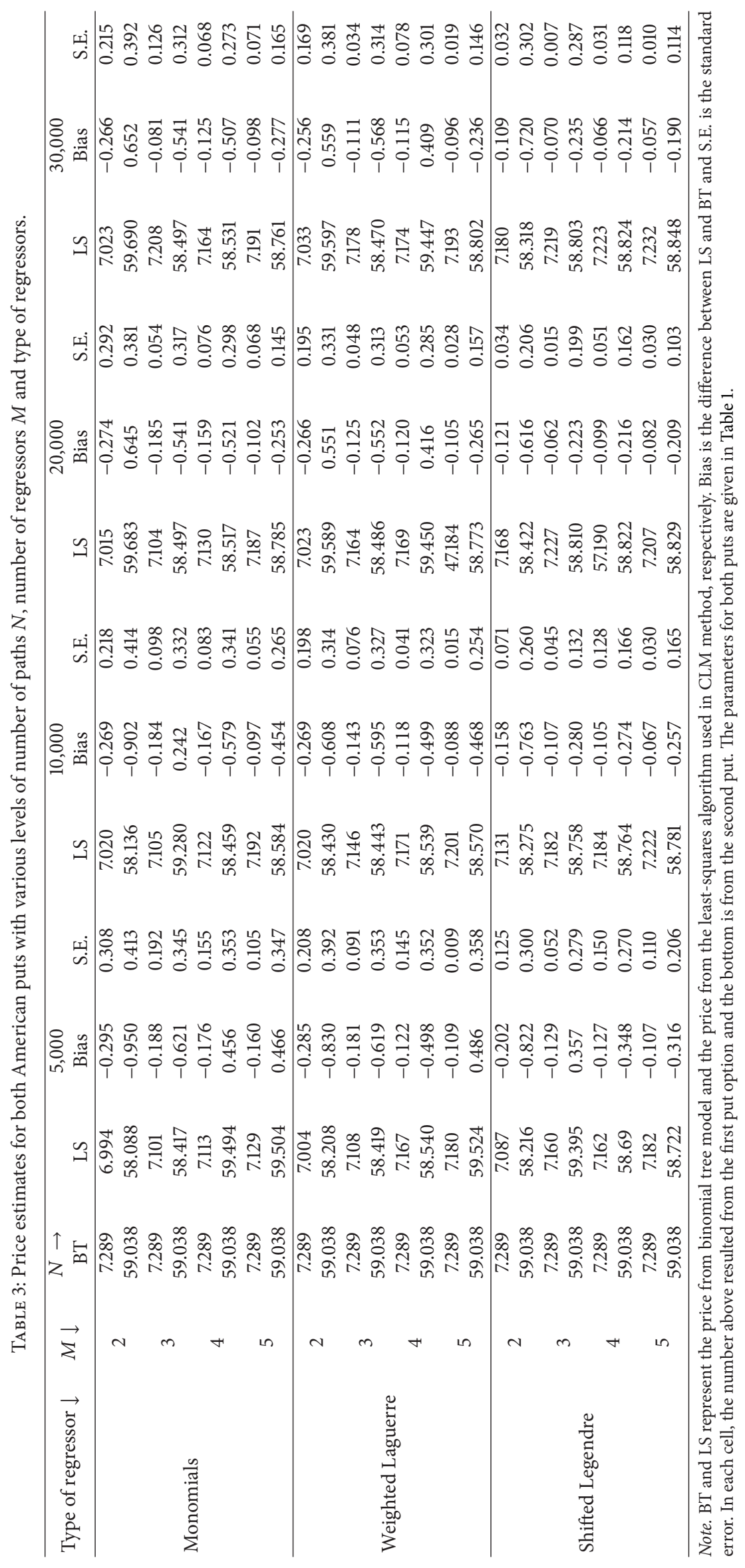


least-squares algorithm provides lower bounds for American puts. Another obvious thing to note is that the bias for the second American put option is larger than the corresponding bias for the first put. This may be due to the fact that the basis point (i.e., the "true" price from BT) of the second put option is 59.038, which is much greater than that of the first put, 7.298 .

Second, for all the types of regressor, the absolute value of bias basically decreases with the increase of the number of regressors, as well as the number of simulated paths, and the smallest bias even reaches -0.057 for the first option and -0.190 for the second one by using the type of Shifted Legendre polynomials. It means that the convergence could be guaranteed when the numbers of regressors and paths are increased. Meanwhile, the standard error has a similar tendency with the bias when the numbers of regressors and paths increase. The findings are consistent with the convergence theorems presented earlier and we can image that the bias potentially vanishes as both numbers of regressors and simulated paths increase. It should also be noted that for each of type of regressor, the bias is overall larger in the case of $M=2$ or 3 than that in the case of $M=4$ or 5 , but there is no big difference between the cases of $M=4,5$. With considering the computational cost, this suggests that a reasonable number of regressors may be $M=4$ when empirically implementing the CLM method in the next section.

Third, based on the number of simulated paths, one observation can be obtained. Although the bias for both options, as mentioned above, decreases with the number of paths given the number of regressors, it is easy to observe that no significant difference is found among four cases of $N=$ $5000,10000,20000$, and 30000 , especially when the number of paths $N$ is greater than 5000. Again, considering the computational burden in the empirical pricing investigation, we generate 10000 underlying price paths.

Fourth, with regard to the type of regressor, from the bias and standard error, using weighted Laguerre polynomials has a little bit advantage over the Monomials polynomials. But choosing the Shifted Legendre polynomials as regressors obviously outperforms other choices. It is not difficult to find that the standard errors become smaller when the Shifted Legendre polynomials are used, especially for the large number of regressors and only two exceptions appear among 32 cases when comparing the Shifted Legendre with weighted Laguerre polynomials. Besides, given the number of regressors, overall the bias from the Shifted Legendre polynomials is also smaller compared with those from two other polynomials. Therefore, the Shifted Legendre polynomials seem to be a better choice than either of the other types of polynomials and this encourages us to use this type of polynomials as the regressors in the empirical test of CLM.

In summary, through the comparison of bias and standard errors, it suggests that the algorithm in CLM method is able to ensure that the price estimate can converge to the actual option values at a rate given in the convergence theorems. Meanwhile, we also show that it is better to choose the Shifted Legendre polynomial as the specification of the cross-sectional regressors. In addition, using four numbers of regressors and 10000 simulated underlying paths is more appropriate.

\section{Empirical Comparison Based on IBM and OEX Puts}

To investigate the performance of canonical least-squares Monte Carlo (CLM) valuation approach, this section makes several comparisons using IBM put option data and OEX put data. The empirical investigation involves three pricing methods including CLM method and two benchmark methods of Crank-Nicolson finite difference (FD) and binomial tree (BT).

One should note that it is impossible to empirically compare the proposed CLM with the pure least-squares Monte Carlo of Longstaff-Schwartz (LSM, see [2]) using actual market data; the reason is as follows. Although the LSM method is applicable to American options, prior to estimating the option price via LSM method, it requires a sample of already simulated risk-neutral paths of the underlying price. In other words, with the market data, one has to take some technique to generate the price paths for the underlying asset and then he/she can use the least-squares algorithm to estimate the price American option. It is exactly due to this that CLM uses the canonical method (Stutzer, [1]) to obtain the risk-neutral distribution with which the riskneutral underlying paths are generated and then prices the American option using the least-squares algorithm.

\subsection{Methodologies}

4.1.1. CLM and Computational Details. The implementation of CLM method is fully detailed in Section 2 and according to the numerical assessing in Section 3.2, we use the first three terms of the Shifted Legendre polynomial plus a constant term as basis functions. Recall that $M=0.4 N^{1 / 4}$ in Section 3.1; the number of underlying paths used in this empirical study is 10,000 .

For each reported price in this study, three independent Monte Carlo runs are carried out and the resultant prices averaged. In every Monte Carlo run, 10,000 paths are simulated, with each path made up of one-day gross returns sampled from the empirically estimated risk-neutral distribution derived in CLM.

Each path is further divided into a number of possible exercising steps according to the following rule: if the days to maturity are less than 50, the size of the exercising step is one day; otherwise, the number of days in one step is the integer part of the number of days to maturity divided by 50 . Note that the last step may cover fewer days than all the other steps, and thus it has to be handled separately.

As for the treatment of interest rate and the dividends, Section 4.2 presents a specified description.

4.1.2. FD and Computational Details. Finite difference methods price options by solving the Black-Scholes (BS) partial differential equation numerically (see Hull [21]). At least three variations, namely, explicit finite difference, implicit 
finite difference, and Crank-Nicolson finite difference, are available. By averaging the implicit and explicit methods, Crank-Nicolson converges faster, and due to this, the CrankNicolson finite difference (FD) method is chosen in this study.

Follow [21] here. Let $Z=\ln S$. The well-known BS equation is then as follows:

$$
\begin{gathered}
\frac{\partial f}{\partial t}+v \frac{\partial f}{\partial Z}+\frac{1}{2} \sigma^{2} \frac{\partial^{2} f}{\partial Z^{2}}=r f \\
v=r-q-0.5 \sigma^{2}
\end{gathered}
$$

where $r$ is the risk-free rate, $q$ the dividend yield, $\sigma$ the volatility, and $f$ the value of a derivative.

The time to maturity $T$ is divided into $N$ intervals of length $\Delta t=T / N$. A sufficiently large $Z, Z_{\max }$, is chosen and define $\Delta Z=Z_{\max } / M$, where $M+1$ is the number of prices to be used. Then one has the following finite difference equation (see [22]):

$$
\begin{array}{r}
p_{u}\left(f_{i, j+1}+f_{i+1, j+1}\right)+p_{m}\left(f_{i, j}+f_{i+1, j}\right) \\
+p_{d}\left(f_{i, j-1}+f_{i+1, j-1}\right)=2 f_{i+1, j} \\
p_{u}=-\frac{\Delta t}{4}\left(\frac{\sigma^{2}}{\Delta Z^{2}}+\frac{v}{\Delta Z}\right), \\
p_{m}=1+\frac{\Delta t}{2}\left(\frac{\sigma^{2}}{\Delta Z^{2}}+r\right), \\
p_{d}=-\frac{\Delta t}{4}\left(\frac{\sigma^{2}}{\Delta Z^{2}}-\frac{v}{\Delta Z}\right),
\end{array}
$$

where $i$ indicates time steps and $j$ price points. The system of (18) is solved backwards from $T-1$ to the valuation date $t_{0}$, while taking suitable boundary conditions into consideration. Values of $f_{i, j}$ obtained at each time step are updated by checking against early exercise (if applicable).

The price of a derivative is obtained by interpolating $f_{0, j}$, given $S_{0}$ at $t_{0}$.

Here FD, applicable to American options, is utilized as a benchmark for CLM. For any meaningful comparison, pricing of different methods should be done with exactly the same input information. Since the same set of daily gross returns is used to estimate the historical volatility used in FD as well as the risk-neutral measure (3) used in CLM, FD with historical volatility solving the BS equation is with no doubt the right benchmark for CLM in specific or any new pricing method in general.

The computations of FD use as default a time step of one day and a stock price spacing of $\Delta Z=\sigma \sqrt{3 \Delta t}$. (This is the convergence condition for the explicit finite difference method (Clewlow and Strickland [22]). For simplicity of programming, however, this price spacing is used for all finite difference methods, including Crank-Nicolson, for which the condition is of course unnecessary.) Thus the optimal early exercising is checked daily. Within each day, the diffusion is repeated for 125 to 6 times, which is the integer part of 2000 divided by the number of days to maturity (between 16 and
300 days). A big number of 2000 is chosen to ensure the convergence of FD. Again, the issues on the interest rate and dividends are discussed in Section 4.2.

4.1.3. BT and Computational Details. In addition to FD method, this study uses another widely used benchmark of the binomial tree method (Cox et al. [23]).

In this binomial model, we use the up and down step sizes calculated by $u=e^{\sigma \sqrt{\Delta t}}$ and $u=e^{-\sigma \sqrt{\Delta t}}$ where $\sigma$ refers to the historical volatility estimated using the observed closing prices of the underlying asset, and $\Delta t$ is the length of time interval of one step. Considering the computational cost but without loss of the convergence, we use 4,000-step tree for each option pricing.

Due to the dividend payments, the pricing issue becomes trickier. With a known dividend yield $q$, if the time point is prior to the exdividend date, keep the usual underlying price at the corresponding node on the tree; otherwise the usual price is multiplied by a factor $(1-q)$. With a known cash dividend, on the exdividend date, the underlying price is reduced by the amount of the dividend payment. Please refer to Hull [20, pages 417-418] for full details.

4.2. Treatment of Dividends and Risk-Free Interest Rate. The dividend paid by the underlying asset of a derivative may be a complex problem, due to tax issues and the often stochastic nature of the dividend payment (either the amount or timing of the payment or both). To simplify this matter, several common assumptions are usually made in pricing exchangetraded options, which typically last for less than one year. For example, it is assumed that on the exdividend date the stock price is reduced by the amount of the dividend payment (see Hull [21]). Note that the resultant stock price will be called the dividend-adjusted stock price hereafter.

As of September 30, 2009, eighty-nine stocks in the S\&P 100 Index paid dividends (data available from CBOE). For example, during the period of July 2008 through January 2009 covering the most dramatic part of the US financial crisis, those dividend payments amount to an annualized continuous yield of about 3\%, which are consistently higher than the one-year risk-free interest rates over the same period. Clearly, the price of a put would be underestimated if dividends were not taken into consideration. Thus for a credible empirical test, the effect of dividends from the S\&P 100 Index should not be ignored.

In derivatives pricing, a stock index is typically assumed to be an underlying asset paying a known constant yield (see [21]). Under the Black-Scholes framework, the price process of the underlying is further assumed to follow a geometric Brownian motion (GBM). Together, these assumptions lead to the well-known BS equation (17), which can be solved numerically by FD. CLM does not have to assume a particular form for the underlying process, but instead simulates paths for the price of the underlying asset directly using the empirically estimated risk-neutral distribution (3). With riskneutral paths given, the effect of a continuous dividend yield can then be handled trivially: the risk-neutral price for the 
TABLE 4: Data description of OEX and IBM puts.

\begin{tabular}{|c|c|c|c|c|c|}
\hline & & Mean & Standard deviation & Minimum & Maximum \\
\hline \multirow{4}{*}{ OEX } & Market price & 55.72 & 58.46 & 0.05 & 412.00 \\
\hline & Days to maturity & 69.25 & 58.68 & 16 & 360 \\
\hline & Moneyness & 1.08 & 0.31 & 0.41 & 2.23 \\
\hline & Number of puts & 208,130 & & & \\
\hline \multirow{4}{*}{ IBM } & Market price & 16.82 & 21.25 & 0.05 & 104.33 \\
\hline & Days to maturity & 119.33 & 70.67 & 16 & 357 \\
\hline & Moneyness & 1.01 & 0.29 & 0.44 & 2.13 \\
\hline & Number of puts & 72,421 & & & \\
\hline
\end{tabular}

Note. In this table, market price refers to the closing price of the underlying, that is, the OEX index price for OEX puts and IBM stock price for IBM puts, and each option price is considered as the midvalue of bid-ask quotations. Moneyness is defined as strike price divided by underlying price.

first day along a path is reduced by a factor of $\exp (q / 365)$, price for the second day by $\exp (2 q / 365)$, and so on.

The IBM stock instead pays regular quarterly cash dividends, which makes the dividend actually more difficult to handle than the dividend yield. To simplify the matter, it is assumed that the amount and timing of the dividend are both known in advance when pricing IBM options. Finite difference for IBM options now solves (17) with $q$ being set to zero. Since the stock price points are fixed in the backwards induction of $\mathrm{FD}$, the option value on the exdividend date is adjusted downward by quadratic interpolation to reflect the effect of dividend payment. This then corresponds to the option value for the dividend-adjusted stock price. If the dividend-adjusted stock price is less than the second lowest stock price on the grid, however, the corresponding option value is reset to that of the lowest stock price in the grid, and no further interpolation is done.

With CLM for IBM options that takes cash dividends into consideration on the other hand, the simulated riskneutral price for every path on exdividend date is reduced exactly by the dividend amount. If the resultant dividendadjusted stock price turns out to be less than zero, it is reset to zero. Regarding BT, this issue can be settled according to the discussion earlier in Section 4.1.3.

As for the risk-free interest rate, unlike what is done in many previous studies, the risk-free interest rates for each valuation date are obtained from the daily US Treasury yield curve (from one month to 30 years). The yield curve was obtained directly from the Web site of the US Department of the Treasury (http://www.ustreas.gov/offices/domesticfinance/debt-management/interest-rate/). If a maturity does not fall on any date given by the yield curve, the corresponding risk-free rate is interpolated linearly from the yield curve. From the valuation date to maturity, the continuously compounded risk-free interest rate is then assumed to be flat.

4.3. Data Description. The American-style S\&P 100 Index (OEX) puts and the equity puts on the IBM common stock (traded on NYSE with the symbol IBM) are chosen for this empirical study. The daily data (including last ask and bid prices) of OEX and IBM put options are collected for the period January 2, 2006, through October 31, 2012 (this not easily obtained data acquired from a database was kindly provided by one friend in UNSW, Australia.), with a total of 1721 trading days. The daily prices for both S\&P 100 Index and IBM stock are downloaded from the CBOE (http://www.cboe.com/DelayedQuote/QuoteTableDownload.aspx), and the closing price is treated as the underlying price for S\&P 100 Index as well as IBM stock.

Only puts with a maturity of 16 (effectively, the majority of puts expiring in the current month are excluded) to 360 days are included in this study. According to Coval and Shumway [15], the average of bid and ask prices of options is treated as its market price, and market prices below $\$ 0.05$ are discarded. The prices of put options should theoretically increase with strike prices. Data violating this rule are discarded. For example, on July 30, 2008, the OEX puts expiring in August 2008 show the following average quote (strike): 0.525 (535), 0.325 (530), and 0.35 (525). So 0.325 is discarded. Once these filter rules are applied, we are left with the total valid number of market prices for OEX puts turns being 208, 130 and for IBM puts 72,421 . Table 4 briefly describes the filtered data. In addition, options with negative implied volatility are removed from the sample ("negative" here refers to the situation when a numerical search failed to find a positive solution for the implied volatility).

Both CLM and the estimation of the historical volatility for $\mathrm{FD}$ and $\mathrm{BT}$ use the observed closing prices of the underlying asset as inputs. It is not obvious unfortunately how many observations will be included in either case; intuitively, one should compare the two cases using the same set of inputs. On the other hand, one could also argue that while CLM may need more data points due to its statistical nature, relatively fewer closing prices will be used for the estimation of the historical volatility (see [21]). To be conservative then, two sets of pricing were carried out for comparison for any trading date; one uses the immediate previous 130 closing prices (roughly half a year in calendar days) of the underlying asset as inputs and the other 260 prices.

For the S\&P 100 Index, a continuous constant annual dividend yield for each valuation date is assumed, which is between 2.18 and 3.92 percent, with an average of 3.01\%, over the whole period. For IBM for the period covered, depending on the valuation date, the dividend payment dates are assumed to be on February 6, 2006, until August 6, 2012 , with one payment every quarter, and the corresponding quarterly dividends are actually $\$ 0.5$ according to the data. 
TABLE 5: OEX put prices computed using the previous 130 daily closing prices of the S\&P 100 Index.

\begin{tabular}{|c|c|c|c|c|c|c|c|c|c|c|}
\hline \multirow{2}{*}{ Moneyness $\downarrow$} & \multirow{2}{*}{$\begin{array}{l}\text { Maturity } \\
\text { Method }\end{array}$} & \multicolumn{3}{|c|}{ Short } & \multicolumn{3}{|c|}{ Medium } & \multicolumn{3}{|c|}{ Long } \\
\hline & & CLM & $\mathrm{FD}$ & BT & CLM & FD & BT & CLM & $\mathrm{FD}$ & BT \\
\hline \multirow{4}{*}{ DOTM } & RMSE & 2.102 & 3.132 & 2.985 & 2.854 & 3.087 & 3.052 & 6.260 & 5.720 & 6.053 \\
\hline & MPE & -0.813 & -1.521 & -1.334 & -0.462 & -1.930 & -1.847 & 3.123 & 1.381 & 2.394 \\
\hline & MAE (\%) & 40.602 & 54.780 & 50.641 & 36.230 & 44.035 & 41.875 & 36.372 & 35.322 & 36.024 \\
\hline & Number of puts & & 30113 & & & 19535 & & & 3721 & \\
\hline \multirow{4}{*}{ OTM } & RMSE & 4.208 & 4.970 & 5.203 & 5.603 & 4.282 & 5.203 & 13.483 & 10.444 & 12.356 \\
\hline & MPE & 0.029 & -2.030 & 0.093 & 2.147 & -1.179 & -2.305 & 10.081 & 5.743 & 8.215 \\
\hline & MAE (\%) & 17.625 & 26.530 & 28.030 & 17.523 & 17.738 & 16.936 & 22.873 & 20.331 & 21.658 \\
\hline & Number of puts & & 26243 & & & 15392 & & & 3630 & \\
\hline \multirow{4}{*}{ ITM } & RMSE & 4.008 & 4.520 & 4.238 & 8.053 & 5.052 & 5.965 & 17.073 & 13.234 & 14.354 \\
\hline & MPE & 1.139 & 1.183 & 1.256 & 5.617 & 2.481 & 3.250 & 14.651 & 10.213 & 11.032 \\
\hline & MAE (\%) & 9.655 & 13.284 & 12.359 & 11.403 & 6.278 & 8.216 & 20.393 & 13.421 & 18.310 \\
\hline & Number of puts & & 26066 & & & 14624 & & & 3692 & \\
\hline \multirow{4}{*}{ DITM } & RMSE & 2.030 & 2.134 & 2.165 & 6.265 & 4.753 & 5.034 & 22.680 & 15.360 & 18.235 \\
\hline & MPE & 0.342 & -0.043 & -0.061 & 4.389 & 2.793 & 3.240 & 18.019 & 12.282 & 15.201 \\
\hline & MAE (\%) & 1.356 & 1.450 & 1.531 & 4.357 & 2.942 & 4.023 & 11.920 & 8.403 & 10.326 \\
\hline & Number of puts & & 35458 & & & 22315 & & & 7341 & \\
\hline
\end{tabular}

Note. This table reports the empirical results for OEX puts using the previous 130 daily closing prices as inputs. Each cell represents a particular combination of moneyness and time to maturity. The last row reports the number of options with corresponding combination. The remaining rows show the error statistic results of RMSE, MPE, and MAE.

4.4. Empirical Results. The results of pricing are summarized and compared using the twelve categories of moneyness and maturity suggested by Barone-Adesi et al. [24]. Specifically, moneyness is divided into four segments: less than 0.85 (deep out-of-the-money or DOTM), 0.85 to 1.00 (out-of-themoney or OTM), 1.00 to 1.15 (in-the-money or ITM), and more than 1.15 (deep in-the-money or DITM). Maturity is classified into three groups: 16 to 60 days (Short), 61 to 160 days (Medium), or 161 to 360 days (Long). Hereafter, the categories of moneyness and maturity will be referred to as OTMShort, as an example, for the category of puts with moneyness between 0.85 and 1.00 and maturity between 16 and 60 days.

Three statistic measures, namely, RMSE, MPE, and MAE, are utilized for pricing comparison, which are again similar to [24]. RMSE or the dollar root-mean-square error is the square root of the averaged squared deviations between calculated prices $p_{j}^{c}$ and market prices $p_{j}^{m}, \sqrt{(1 / N) \sum_{j=1}^{N}\left(p_{j}^{c}-p_{j}^{m}\right)^{2}}$. MPE or the dollar mean pricing error is the average of the pricing errors, $(1 / N) \sum_{j=1}^{N}\left(p_{j}^{c}-p_{j}^{m}\right)$. MAE or the percentage mean absolute error is the average of the absolute pricing errors expressed in percentage, $(1 / N) \sum_{j=1}^{N}\left|p_{j}^{c} / p_{j}^{m}-1.0\right|$. From a trader's point of view, MAE is probably the best indicator for the overall accuracy of a pricing model; it then seems reasonable to prefer a pricing model with a smaller overall percentage mean absolute error.

Table 5 shows the summarized result for OEX put prices using the immediate previous 130 daily closing prices of the S\&P 100 Index as inputs. From the number of each combination, although the four categories with all the longest maturities have fewer puts traded, even the smallest number among the four categories, 3630 for OTM-Long, it is still adequate for drawing meaningful statistical conclusions.
In terms of RMSE, FD overall performs better, especially for ITM-Medium, DITM-Medium, and all the four longest maturity categories, but BT is comparable to CLM. With respect to MPE, CLM outperforms FD and BT for DOTMShort, DOTM-Medium, and OTM-Short but underperforms for all the DITM, and the four longest maturity categories. From the most important MAE measure, CLM outperforms FD in MAE for DOTM-Short and DOTM-Medium but does poorly for ITM-Short, ITM-Medium, DITM-Medium, and all the four longest maturity categories. Overall, CLM underperforms FD or BT. The percentage pricing errors from all the methods are not small with an exception of DITM, especially for the case of OTM; the MAE errors are even up to $54.78 \%$. Thus, it is not appropriate to use only 130 closing prices for CLM, FD, and BT.

OEX put prices using the previous 260 daily closing prices of the S\&P 100 Index as inputs are shown in Table 6. CLM does poorly in RMSE for three out of the four longest maturity categories. In terms of MPE, CLM outperforms FD and BT for all three DOTM cases but underperforms either BT or FD for four combinations of moneyness and maturity in other nine combinations. With respect to MAE, CLM outperforms FD in most categories but three categories of OTM-Long, ITMLong, and DITM-Long. Meanwhile, CLM outperforms BT for all six out-of-money categories and ITM-Medium, DITMShort, and DITM-Medium categories.

Going from 130 to 260 days, CLM yields better statistics in 33 combinations of moneyness and maturity out of 36 combinations for three measures, while the situations for FD and BT are mixed. For FD, it overall improves the pricing results but produces worse results when the option is DOTM-Medium, DOTM-Long, OTM-Short, or OTMMedium, measured by the most important indicator MAE. 
TABLE 6: OEX put prices computed using the previous 260 daily closing prices of the S\&P 100 Index.

\begin{tabular}{|c|c|c|c|c|c|c|c|c|c|c|}
\hline \multirow{2}{*}{ Moneyness $\downarrow$} & \multirow{2}{*}{$\begin{array}{l}\text { Maturity } \\
\text { Method }\end{array}$} & \multicolumn{3}{|c|}{ Short } & \multicolumn{3}{|c|}{ Medium } & \multicolumn{3}{|c|}{ Long } \\
\hline & & CLM & $\mathrm{FD}$ & $\mathrm{BT}$ & CLM & FD & BT & CLM & $\mathrm{FD}$ & BT \\
\hline \multirow{4}{*}{ DOTM } & RMSE & 2.273 & 2.719 & 2.756 & 3.135 & 4.433 & 4.398 & 3.261 & 4.353 & 4.686 \\
\hline & MPE & -1.259 & -1.801 & -2.047 & -2.137 & -3.126 & -3.043 & -0.790 & -3.202 & -2.189 \\
\hline & MAE (\%) & 38.729 & 46.864 & 45.730 & 33.700 & 48.657 & 46.497 & 20.886 & 36.821 & 37.523 \\
\hline & Number of puts & & 30113 & & & 19535 & & & 3721 & \\
\hline \multirow{4}{*}{ OTM } & RMSE & 3.246 & 4.750 & 4.983 & 3.139 & 5.769 & 6.690 & 4.920 & 4.939 & 6.851 \\
\hline & MPE & -2.171 & -3.731 & -1.601 & -1.445 & -3.732 & -4.858 & 3.392 & -2.135 & 0.337 \\
\hline & MAE (\%) & 22.300 & 33.979 & 35.479 & 23.264 & 22.936 & 22.134 & 13.171 & 12.950 & 14.277 \\
\hline & Number of puts & & 26243 & & & 15392 & & & 3630 & \\
\hline \multirow{4}{*}{ ITM } & RMSE & 2.509 & 3.341 & 3.059 & 3.269 & 3.956 & 4.869 & 6.754 & 5.018 & 6.138 \\
\hline & MPE & -0.266 & -1.674 & -1.601 & 1.221 & -0.930 & -0.161 & 4.661 & 2.160 & 1.979 \\
\hline & MAE (\%) & 5.893 & 6.079 & 5.154 & 5.750 & 5.793 & 7.731 & 10.857 & 6.550 & 9.439 \\
\hline & Number of puts & & 26066 & & & 14624 & & & 3692 & \\
\hline \multirow{4}{*}{ DITM } & RMSE & 1.926 & 2.192 & 2.223 & 2.225 & 2.404 & 2.685 & 9.713 & 7.389 & 8.264 \\
\hline & MPE & -0.778 & -0.776 & -0.794 & 0.388 & 0.452 & 0.899 & 7.732 & 5.886 & 8.805 \\
\hline & MAE (\%) & 1.071 & 1.179 & 1.260 & 1.243 & 1.429 & 2.510 & 4.320 & 3.786 & 3.809 \\
\hline & Number of puts & & 35458 & & & 22315 & & & 7341 & \\
\hline
\end{tabular}

Note. This table reports the empirical results for OEX puts using the previous 260 daily closing prices as inputs. Each cell represents a particular combination of moneyness and time to maturity. The last row reports the number of options with corresponding combination. The remaining rows show the error statistic results of RMSE, MPE, and MAE.

The situation for BT is similar to that for FD. Further, CLM with 260 days provides better or comparable pricing performance in terms of MAE in almost all the categories of moneyness and maturity with an exception of OTM-Short and is reasonably accurate (MAE under $10 \%$ ) for all six in-themoney categories, while neither of the situations for FD and $\mathrm{BT}$ is the case. In addition, BT is comparable to FD and they make no big difference. In conclusion, CLM works better with 260 closing prices and outperforms FD as well as BT overall in pricing OEX puts.

Three more observations can be made. First, for all three methods in most of the categories, the pricing errors increase with the decrease of moneyness as well as maturity. Second, for ITM-Long and DITM-Long, CLM, FD, and BT are fairly accurate and FD slightly outperforms CLM. These results are not surprising however, since the market seems to have been put a premium on the out-of-money puts ever since the 1987 market crash, but not on the in-the-money or longer maturity options according to Black-Scholes. MAE errors from three methods are quite large for most of six out-ofthe-money categories, which is also consistent with previous studies and the well-known phenomenon of volatility smile or smirk (Hull, [21]).

Next, turn to the results for IBM puts. The IBM puts are distributed more evenly than OEX puts among the twelve categories, with 3527 for OTM-Long being the lowest. Table 7 shows the summarized result for put prices using the previous 130 daily closing prices of the IBM common stock as inputs. RMSE errors are comparable among CLM, FD, and BT, so are MPE errors. In terms of MAE, similar to the situation for OEX, three methods yield lager errors for all the six out-of-money categories, but apparently CLM dominantly outperforms both FD and BT in these cases. When options are in-the-money, each of the methods produces a low MAE error, especially for the puts being DITM.

IBM put prices using the previous 260 daily closing prices of the corresponding common stock as inputs are shown in Table 8. CLM is totally superior to BT for RMSE errors and performs better than FD since it underperforms only in the cases of ITM-Long and DITM-Medium out of all twelve categories. In terms of MAE, CLM outperforms FD and BT once again for most categories; it only slightly underperforms both FD and BT for ITM-Long and DITM-Long put options and also underperforms FD for DTIM-Medium puts.

From Tables 7 and 8, it is not difficult to observe that CLM and FD with either 130 or 260 closing prices produce reasonably accurate prices (MAE is around or under $10 \%$ ) for all the six in-the-money categories of IBM puts; this is unlike the situation for OEX puts. Further, the pricing results are overall improved by using 260 daily prices instead of 130 data for both IBM and OEX puts.

In summary, even though CLM, FD, and BT generate quite large pricing errors for the six out-of-the-money categories, CLM outperforms both FD and BT in pricing overall with 260 closing prices of the underlying as inputs for both the American-style S\&P 100 Index puts and IBM puts. This naturally leads to the following two inferences, considering that CLM and Black-Scholes were shown previously to yield nearly identical prices for GBM (see [3]). First, the empirically observed underlying prices for both the S\&P 100 Index and the IBM stock did not follow GBM processes. Second, CLM with enough prices as inputs can handle nonGBM processes better than Black-Scholes in terms of options pricing. 
TABLE 7: IBM put prices computed using the previous 130 daily closing prices of the IBM common stock.

\begin{tabular}{|c|c|c|c|c|c|c|c|c|c|c|}
\hline \multirow{2}{*}{ Moneyness $\downarrow$} & \multirow{2}{*}{$\begin{array}{l}\text { Maturity } \\
\text { Method }\end{array}$} & \multicolumn{3}{|c|}{ Short } & \multicolumn{3}{|c|}{ Medium } & \multicolumn{3}{|c|}{ Long } \\
\hline & & CLM & $\mathrm{FD}$ & $\mathrm{BT}$ & CLM & FD & BT & CLM & $\mathrm{FD}$ & BT \\
\hline \multirow{4}{*}{ DOTM } & RMSE & 0.523 & 0.582 & 0.576 & 0.785 & 1.076 & 0.964 & 0.946 & 1.201 & 1.352 \\
\hline & MPE & -0.343 & -0.405 & -0.386 & -0.601 & -0.837 & -0.812 & -0.346 & -0.978 & -1.025 \\
\hline & MAE (\%) & 50.542 & 61.532 & 58.125 & 47.394 & 57.690 & 55.328 & 41.266 & 55.452 & 57.023 \\
\hline & Number of puts & & 6156 & & & 7852 & & & 5230 & \\
\hline \multirow{4}{*}{ OTM } & RMSE & 1.050 & 1.281 & 1.303 & 1.185 & 1.788 & 1.536 & 1.786 & 1.929 & 1.953 \\
\hline & MPE & -0.492 & -0.935 & -0.985 & -0.575 & -1.475 & -1.432 & 0.049 & -1.340 & -1.029 \\
\hline & MAE (\%) & 28.508 & 38.416 & 39.852 & 20.634 & 33.875 & 31.652 & 18.775 & 25.875 & 24.851 \\
\hline & Number of puts & & 4966 & & & 4824 & & & 3527 & \\
\hline \multirow{4}{*}{ ITM } & RMSE & 0.901 & 0.982 & 0.975 & 1.236 & 1.302 & 1.403 & 2.188 & 1.890 & 2.065 \\
\hline & MPE & 0.042 & -0.460 & -0.421 & 0.258 & -0.722 & -0.627 & 0.928 & -0.534 & -0.865 \\
\hline & MAE (\%) & 8.233 & 8.216 & 8.202 & 7.286 & 8.766 & 9.013 & 10.316 & 10.016 & 10.287 \\
\hline & Number of puts & & 5171 & & & 5011 & & & 3676 & \\
\hline \multirow{4}{*}{ DITM } & RMSE & 0.459 & 0.950 & 0.926 & 0.941 & 0.537 & 0.621 & 2.197 & 1.330 & 1.853 \\
\hline & MPE & 0.152 & -0.080 & -0.126 & 0.608 & 0.120 & 0.356 & 1.501 & 0.519 & 1.026 \\
\hline & MAE (\%) & 1.026 & 1.213 & 1.326 & 1.653 & 1.266 & 1.401 & 5.133 & 4.266 & 4.813 \\
\hline & Number of puts & & 8033 & & & 9864 & & & 8111 & \\
\hline
\end{tabular}

Note. This table reports the empirical results for IBM puts using the previous 130 daily closing prices as inputs. Each cell represents a particular combination of moneyness and time to maturity. The last row reports the number of options with corresponding combination. The remaining rows show the error statistic results of RMSE, MPE, and MAE.

TABLE 8: IBM put prices computed using the previous 260 daily closing prices of the IBM common stock.

\begin{tabular}{|c|c|c|c|c|c|c|c|c|c|c|}
\hline \multirow{2}{*}{ Moneyness $\downarrow$} & \multirow{2}{*}{$\begin{array}{l}\text { Maturity } \\
\text { Method }\end{array}$} & \multicolumn{3}{|c|}{ Short } & \multicolumn{3}{|c|}{ Medium } & \multicolumn{3}{|c|}{ Long } \\
\hline & & CLM & FD & BT & CLM & FD & BT & CLM & FD & BT \\
\hline \multirow{4}{*}{ DOTM } & RMSE & 0.564 & 0.615 & 0.609 & 0.980 & 1.189 & 1.077 & 1.001 & 1.478 & 1.629 \\
\hline & MPE & -0.386 & -0.428 & -0.419 & -0.708 & -0.885 & -0.862 & -0.772 & -1.272 & -1.319 \\
\hline & MAE (\%) & 52.825 & 64.323 & 60.354 & 47.885 & 59.715 & 52.893 & 41.816 & 62.002 & 65.120 \\
\hline & Number of puts & & 6156 & & & 7852 & & & 5230 & \\
\hline \multirow{4}{*}{ OTM } & RMSE & 1.064 & 1.336 & 1.358 & 1.346 & 1.917 & 1.666 & 1.185 & 1.915 & 1.939 \\
\hline & MPE & -0.562 & -0.993 & -1.043 & -0.651 & -1.554 & -1.511 & -0.262 & -1.603 & -1.292 \\
\hline & MAE (\%) & 25.841 & 36.074 & 36.359 & 16.701 & 31.191 & 28.563 & 11.458 & 22.366 & 23.765 \\
\hline & Number of puts & & 4966 & & & 4824 & & & 3527 & \\
\hline \multirow{4}{*}{ ITM } & RMSE & 0.888 & 1.011 & 1.004 & 1.230 & 1.416 & 1.517 & 1.511 & 1.474 & 1.649 \\
\hline & MPE & -0.039 & -0.525 & -0.486 & 0.194 & -0.795 & -0.702 & 0.621 & -0.810 & -1.141 \\
\hline & MAE (\%) & 7.856 & 8.407 & 8.324 & 8.169 & 8.364 & 8.536 & 9.391 & 7.216 & 10.137 \\
\hline & Number of puts & & 5171 & & & 5011 & & & 3676 & \\
\hline \multirow{4}{*}{ DITM } & RMSE & 0.401 & 1.189 & 1.165 & 0.607 & 0.523 & 0.617 & 1.310 & 0.771 & 1.293 \\
\hline & MPE & 0.075 & -0.145 & -0.191 & 0.310 & -0.038 & 0.197 & 0.860 & 0.088 & 0.595 \\
\hline & MAE (\%) & 0.751 & 1.279 & 1.028 & 0.828 & 0.817 & 0.905 & 3.216 & 3.025 & 3.149 \\
\hline & Number of puts & & 8033 & & & 9864 & & & 8111 & \\
\hline
\end{tabular}

Note. This table reports the empirical results for IBM puts using the previous 260 daily closing prices as inputs. Each cell represents a particular combination of moneyness and time to maturity. The last row reports the number of options with corresponding combination. The remaining rows show the error statistic results of RMSE, MPE, and MAE.

\section{Conclusions}

Prior to the empirical investigations of the CLM valuation approach, this paper presents the convergence results of CLM and numerically examines the convergence properties. The numerical results show that the algorithm CLM method used is able to ensure the convergence of the estimated price approximating to the actual option value at a rate. A better choice on the type of basis function, the number of regressors, and the number of simulated paths, when implementing CLM method, is also suggested.

Using the American-style S\&P 100 Index puts as a representative for index options with known dividend yields and IBM puts as a representative for equity options with known cash dividends, the paper investigated the empirical performance of the recently proposed CLM method. 
Even though the three methods of CLM, FD, and BT yield reasonably accurate prices for in-the-money OEX and IBM puts (MAE under 10\%), they do not price out-ofthe-money puts accurately. Among the twelve categories of moneyness and maturity, CLM does an overall better job for pricing American puts than the benchmark methods of finite difference and binomial tree, when 260 daily closing prices of the underlying are used as inputs. It is therefore reasonable to conclude that CLM is a useful alternative for pricing American options. Meanwhile, the empirical results also show that the benchmarks of finite difference and binomial tree can price the American put options with a similar accuracy. This finding is consistent with the assertion on the relation between finite difference and binomial tree methods (see Hull [21, pages 443-444] for the relation).

\section{Conflict of Interests}

The authors declare that there is no conflict of interests regarding the publication of this paper.

\section{Acknowledgments}

The authors are truly grateful to the anonymous reviewers for their helpful comments. This work is supported by the research Grant from the National Natural Science Foundation of China (no. 71301132), the Sichuan Provincial Department of Education Research Project (14ZB0448), and the Fundamental Research Funds for the Central Universities (JBK130401).

\section{References}

[1] M. Stutzer, "A simple nonparametric approach to derivative security valuation," The Journal of Finance, vol. 51, pp. 1633-1652, 1996.

[2] F. A. Longstaff and E. S. Schwartz, "Valuing American options by simulation: a simple least-squares approach," Review of Financial Studies, vol. 14, no. 1, pp. 113-147, 2001.

[3] Q. Liu, "Pricing American options by canonical least-squares Monte Carlo," Journal of Futures Markets, vol. 30, no. 2, pp. 175187,2010

[4] J. Alcock and T. Carmichael, "Nonparametric American option pricing," Journal of Futures Markets, vol. 28, no. 8, pp. 717-748, 2008.

[5] X. S. Yu and L. Yang, "Pricing American options using a nonparametric entropy approach," Discrete Dynamics in Nature and Society, vol. 2014, Article ID 369795, 16 pages, 2014.

[6] E. Clément, D. Lamberton, and P. Protter, "An analysis of a least squares regression method for American option pricing," Finance and Stochastics, vol. 6, no. 4, pp. 449-471, 2002.

[7] L. Stentoft, "Convergence of the least squares Monte Carlo approach to American option valuation," Management Science, vol. 50, no. 9, pp. 1193-1203, 2004.

[8] C. R. Harvey and R. E. Whaley, "Dividends and S\&P 100 index option valuation," Journal of Futures Markets, vol. 12, pp. 123137, 1992.

[9] C. R. Harvey and R. E. Whaley, "S\&P 100 index option volatility," Journal of Finance, vol. 46, pp. 1251-1261, 1991.
[10] M. Broadie, J. Detemple, E. Ghysels, and O. Torrés, "American options with stochastic dividends and volatility: a nonparametric investigation," Journal of Econometrics, vol. 94, no. 1-2, pp. 53-92, 2000.

[11] M. Broadie, J. Detemple, E. Ghysels, and O. Torrès, "Nonparametric estimation of American options' exercise boundaries and call prices," Journal of Economic Dynamics \& Control, vol. 24, no. 11-12, pp. 1829-1857, 2000.

[12] S. Poon and F. P. Peter, "Trading volatility spreads: a test of index option market efficiency," European Financial Management, vol. 6, pp. 235-260, 2002.

[13] T. L. Zivney, "The value of early exercise in option prices: an empirical investigation," Journal of Financial and Quantitative Analysis, vol. 26, pp. 129-138, 1991.

[14] F. A. Longstaff, "Option pricing and the martingale restriction," Review of Financial Studies, vol. 8, pp. 1091-1124, 1995.

[15] J. D. Coval and T. Shumway, "Expected option returns," Journal of Finance, vol. 56, no. 3, pp. 983-1009, 2001.

[16] I. Kirgiz, Essays in volatility and stochastic volatility options pricing models [Doctoral dissertation], University of Maryland, College Park, Md, USA, 2001.

[17] D. Kelly and J. Shorish, "Valuing and hedging American put options using neural networks," GSIA Working Papers 8, Carnegie Mellon University, Tepper School of Business, 1994.

[18] L. Stentoft, "American option pricing using GARCH models and the normal inverse Gaussian distribution," Journal of Financial Econometrics, vol. 6, no. 4, pp. 540-582, 2008.

[19] A. Ben-Tal, "The entropic penalty approach to stochastic programming," Mathematics of Operations Research, vol. 10, no. 2, pp. 263-279, 1985.

[20] P. Brandimarte, Numerical Methods in Finance and Economics: A MATLAB Based Introduction, John Wiley \& Sons, New York, NY, USA, 2nd edition, 2006.

[21] J. C. Hull, Options, Futures, and Other Derivatives, Prentice Hall, Upper Saddle River, NJ, USA, 7th edition, 2009.

[22] L. Clewlow and C. Strickland, Implementing Derivatives Models, John Wiley \& Sons, New York, NY, USA, 1998.

[23] J. C. Cox, S. A. Ross, and M. Rubinstein, "Option pricing: a simplified approach," Journal of Financial Economics, vol. 7, no. 3, pp. 229-263, 1979.

[24] G. Barone-Adesi, R. F. Engle, and L. Mancini, "A GARCH option pricing model with filtered historical simulation," Review of Financial Studies, vol. 21, no. 3, pp. 1223-1258, 2008. 


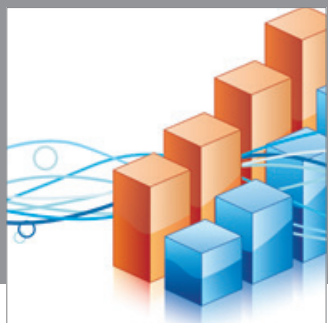

Advances in

Operations Research

mansans

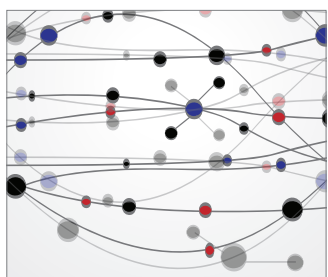

The Scientific World Journal
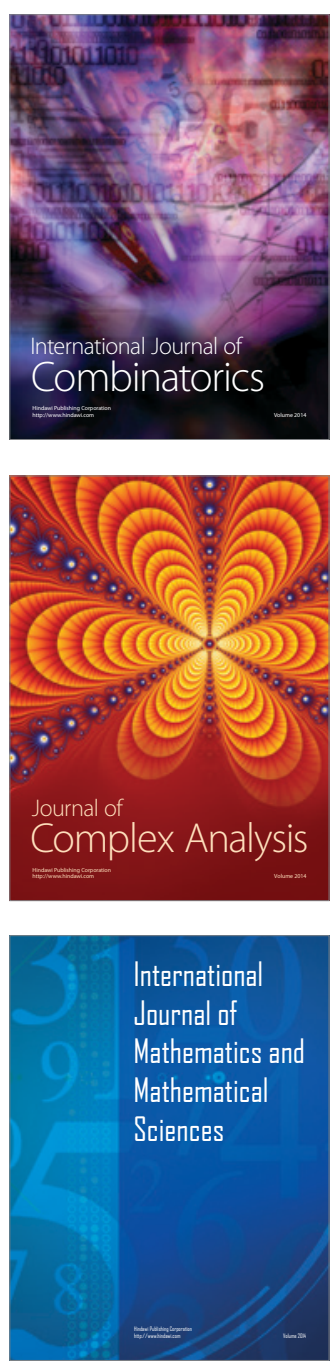
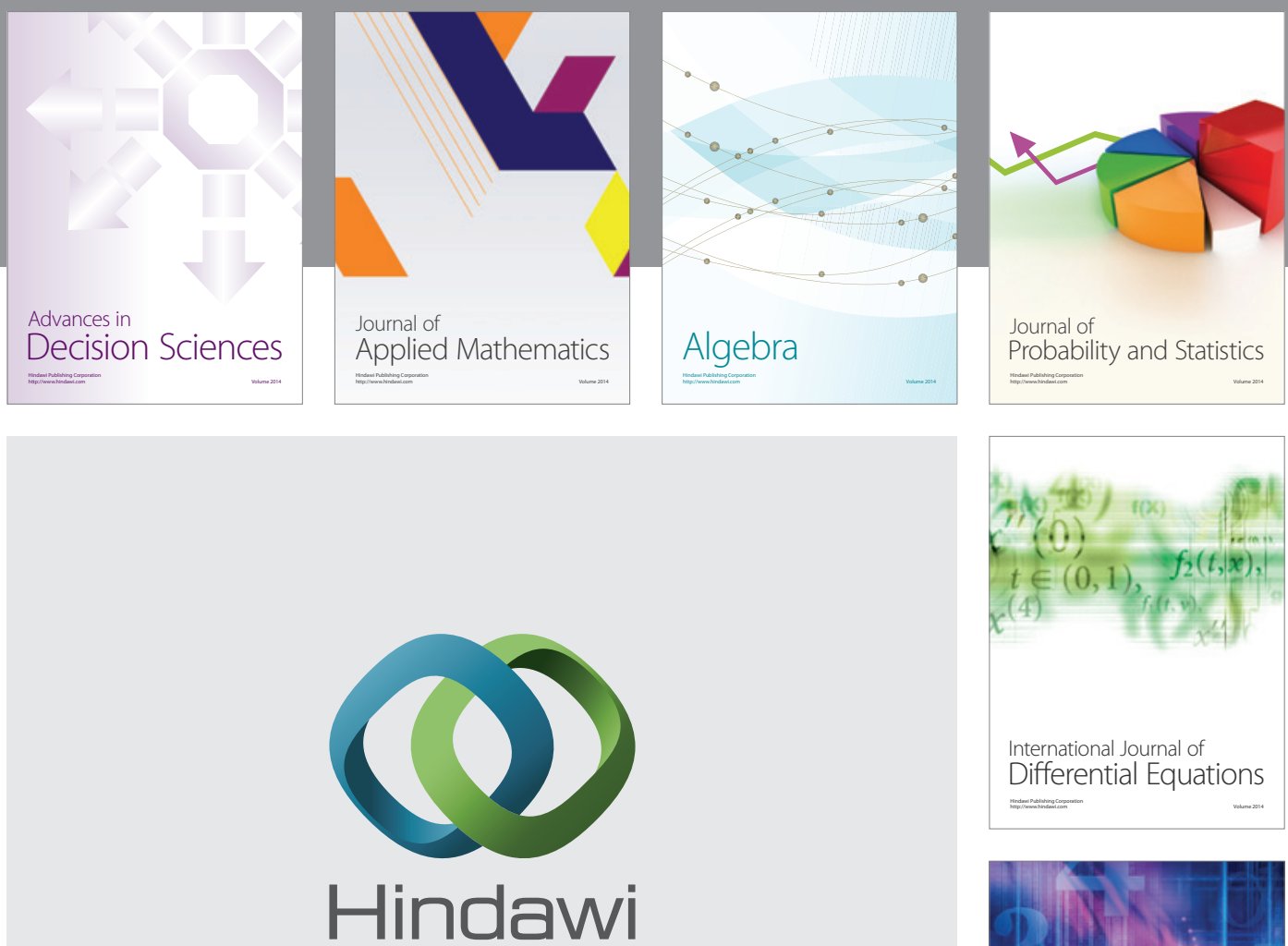

Submit your manuscripts at http://www.hindawi.com
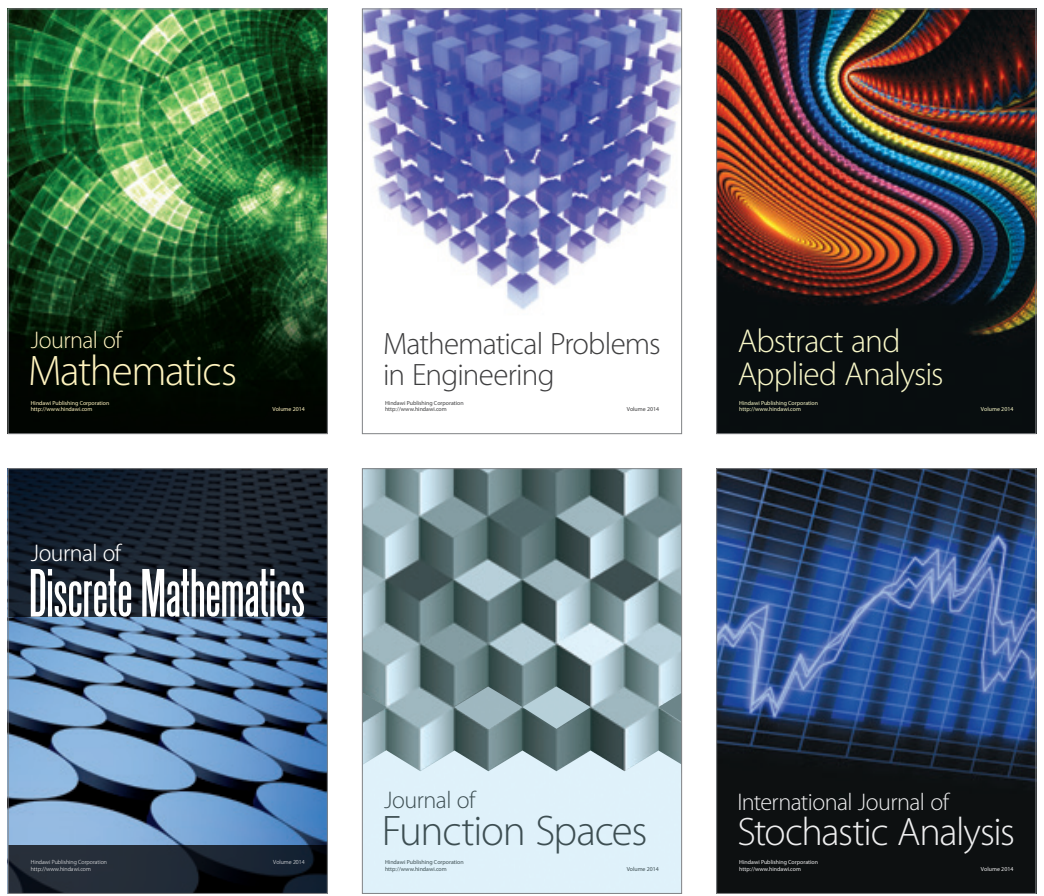

Journal of

Function Spaces

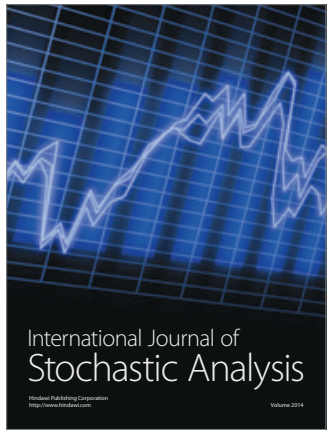

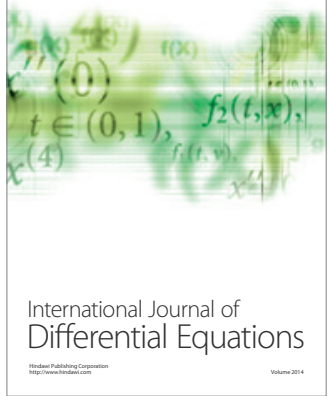
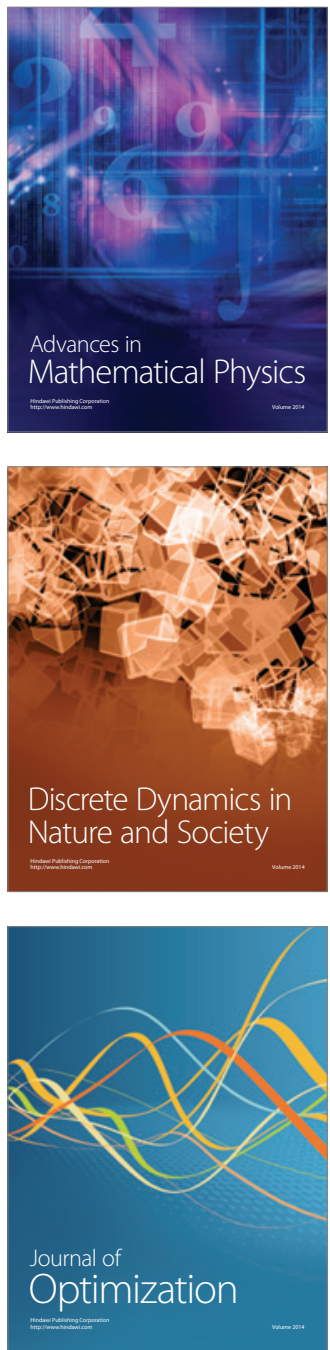